\title{
Avaliação da angulação e inclinação dos elementos dentários em pacientes adultos jovens portadores de fissura transforame incisivo bilateral
}

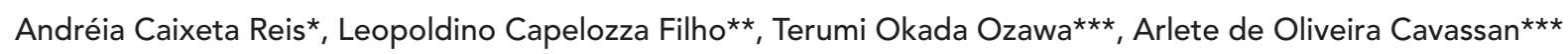

Resumo

Objetivo: desse trabalho foi avaliar e mensurar o posicionamento dentário (angulação e inclinação) em pacientes com fissura transforame incisivo bilateral, no intuito de determinar características comuns a esses pacientes. Metodologia: Para tanto, foram selecionados aleatoriamente, a partir do arquivo do Hospital de Reabilitação de Anomalias Craniofaciais da Universidade de São Paulo (HRAC-USP), trinta pares de modelos de gesso dos arcos dentários de pacientes operados, portadores deste tipo de fissura e em estágio de dentadura permanente, com idades entre 12 e 27 anos. Os modelos foram preparados e as medidas realizadas, baseando-se na metodologia proposta por Andrews ${ }^{1}$. Resultados: Os resultados obtidos foram: 1. Angulação - no arco superior, valores positivos (angulação positiva), com tendência crescente progressiva, de molares para caninos e para os incisivos, valores negativos (contra-angulação). No arco inferior, valores positivos decrescentes de molares para pré-molares e negativos em toda a região anterior; 2 . Inclinação - valores negativos, correspondentes a inclinação palatina ou lingual, para todos os dentes da amostra. Conclusões: Conclui-se que os caninos e incisivos superiores foram os dentes mais afetados pela presença da fissura; o arco inferior manteve valores mais uniformes, devido à menor interferência da fissura; inclinações negativas em todo o arco inferior e apinhamentos anteriores freqüentes sugerem a compensação inferior à maxila atrésica.

Palavras-chave: Ortodontia. Braquetes ortodônticos. Fissura lábio palatal.

\section{INTRODUÇÃO}

O tratamento de pacientes com fissuras lábiopalatais, especialmente os portadores de fissura transforame incisivo bilateral, expressão mais severa desse tipo de deformidade facial, ainda se mantém um desafio ${ }^{10,11}$. As limitações impostas pelo comprometimento anatômico e funcional, potencializadas pelos efeitos deletérios das cirur- gias plásticas primárias sobre o crescimento facial, dificultam sobremaneira o tratamento e sua estabilidade ${ }^{6,21}$. De qualquer maneira, justifica-se o tratamento pelos resultados obtidos, especialmente com as cirurgias plásticas primárias, as quais provocam mudanças de grande impacto estético, funcional e psicossocial ${ }^{15}$.

A literatura é escassa no que se refere à fissura

* Aluna do curso de especialização em Ortodontia Corretiva do Hospital de Reabilitação de Anomalias Craniofaciais da Universidade de São Paulo (HRAC-USP), Bauru/SP.

** Professor Assistente Doutor da Faculdade de Odontologia de Bauru da Universidade de São Paulo (FOB-USP) e Responsável pelo Setor de Ortodontia HRAC-USP, Bauru/SP.

*** Ortodontistas do HRAC-USP, Bauru-SP. 
transforame incisivo bilateral ${ }^{11,17}$. Poucos trabalhos são encontrados relacionados a esse tipo de fissura e, em sua maioria, apresentam inconsistências do tipo: amostras pequenas, protocolos de tratamento variados ${ }^{14}$, incluindo alguns já comprovadamente superados e pouco tempo de acompanhamento longitudinal. Provavelmente isso ocorra em virtude da relativa baixa incidência desse tipo de fissura ${ }^{3,11,15,16,17}$ em relação às demais, aproximadamente $16,5 \%$, e da dificuldade em se eleger um protocolo único para tratá-los, uma vez que as variações individuais são grandes ${ }^{2,21}$ e demandam técnicas diferentes para solucioná-las.

Outro ponto importante a ser destacado é a idade dos pacientes nas amostras, com a maioria dos trabalhos referindo-se às dentaduras decídua e mista ${ }^{3,10}$. Os autores relatam dificuldades em se estudar pacientes na dentadura permanente, já que nesta fase as variáveis aumentam consideravelmente; somam-se às variáveis impostas pela diversidade anatômica da fissura, às impostas pelo desenvolvimento da própria oclusão e cuidados pessoais com alimentação e higiene, dificultando a padronização do estudo ${ }^{10}$.

Além destas limitações, a metodologia da maioria dos trabalhos baseia-se em dados cefalométri$\cos { }^{12,16,19,20,23,24}$, cujos resultados sempre devem ser analisados com parcimônia, devido às limitações deste método.

De qualquer forma, algumas informações sobre os pacientes com fissura transforame incisivo bilateral já são consenso na literatura. Sua morfologia craniofacial é bem descrita: maxila dividida em três segmentos distintos; pré-maxila projetada; redução da columela e aumento de ângulo goníaco. Também encontram-se informações sobre a forma do arco dentário (constricção maxilar mais acentuada na região anterior), causas da atresia maxilar ${ }^{8,13,16}$ e efeito da queiloplastia sobre a pré$\operatorname{maxila}^{13,18,19}$.

A convexidade facial é bastante acentuada em crianças ao nascimento e primeiros anos de vida ${ }^{9,15}$, assim como em pacientes adultos não operados ${ }^{16}$, devido à projeção da pré-maxila. Esta convexidade diminui após a cirurgia do lábio ${ }^{19}$, tendendo a equiparar-se ao normal por volta dos sete anos de idade $^{14}$.

No aspecto dentário, destaca-se a retroinclinação do complexo dento-alveolar superior, tanto em pacientes não operados ${ }^{16}$, como em pacientes operados, nas dentaduras decídua ${ }^{10}$ e permanente ${ }^{19,20}$. Os incisivos inferiores também apresentam normalmente inclinação lingual $1^{4,5,16,22}$.

Neste contexto, inspirado nos estudos de Andrews $^{1}$, idealizador do difundido e eficaz aparelho de Straight Wire, decidiu-se estudar a dentadura permanente em pacientes adultos jovens portadores de fissura transforame incisivo bilateral, no intuito de determinar as variações de posicionamento dentário e, através da individualização, definir a possibilidade de uso do aparelho programado nestes pacientes.

Reconhecendo as dificuldades já descritas pela literatura, esse trabalho tem por objetivo avaliar e mensurar, em modelos de gesso, o posicionamento dentário (angulação e inclinação) em pacientes com fissura transforame incisivo bilateral, no intuito de determinar características comuns a esses pacientes.

\section{MATERIAL E MÉTODOS}

Para a realização desse trabalho foi selecionada uma amostra de 30 pares de modelos de gesso (superior e inferior) de pacientes que cumprissem os seguintes requisitos:

- estarem regularmente matriculados no Hospital de Reabilitação de Anomalias Craniofaciais da Universidade de São Paulo (HRAC-USP);

- serem portadores de fissura transforame incisivo bilateral, sem relatos de síndromes associadas;

- encontrarem-se em estágio de dentadura permanente;

- não terem realizado expansão rápida da maxila (na dentadura permanente) ou qualquer tratamento ortodôntico corretivo;

- não terem realizado enxerto ósseo alveolar e 
- apresentarem oclusão compatível com indicação de tratamento ortodôntico compensatório, ou seja, relativa harmonia entre as bases ósseas maxilares (sem indicação de cirurgia ortognática).

A seleção foi feita aleatoriamente e compreendeu modelos tanto de pacientes que compareceram ao hospital na época da realização do trabalho (2000 a 2002), que preenchiam os requisitos citados acima, quanto modelos dos arquivos do HRAC-USP, mediante estudo de seus registros.

Os materiais utilizados neste estudo foram:

- 30 pares de modelos de gesso;

- compasso de ponta seca;

- gabarito oclusal: placa acrílica rígida, plana, com aproximadamente $2 \mathrm{~mm}$ de espessura, para representar o plano oclusal, sem recorte na região de caninos (Fig. 1-A);

- transferidor com braço de leitura regulável (Fig. 1-B);

Os modelos foram preparados, previamente à realização das medidas, que se basearam na metodologia proposta por Andrews ${ }^{1}$.

Essa preparação consistiu em marcações feitas em cada um dos dentes, do Eixo Vestibular da Coroa Clínica (EVCC) e o seu ponto médio (ponto EV), correspondente ao centro da coroa clínica. Os modelos foram desgastados tanto na base quanto em qualquer outra superfície (regiões retro-molar, vestibular do rebordo alveolar, gengival) que interferisse no contato do gabarito oclusal com as

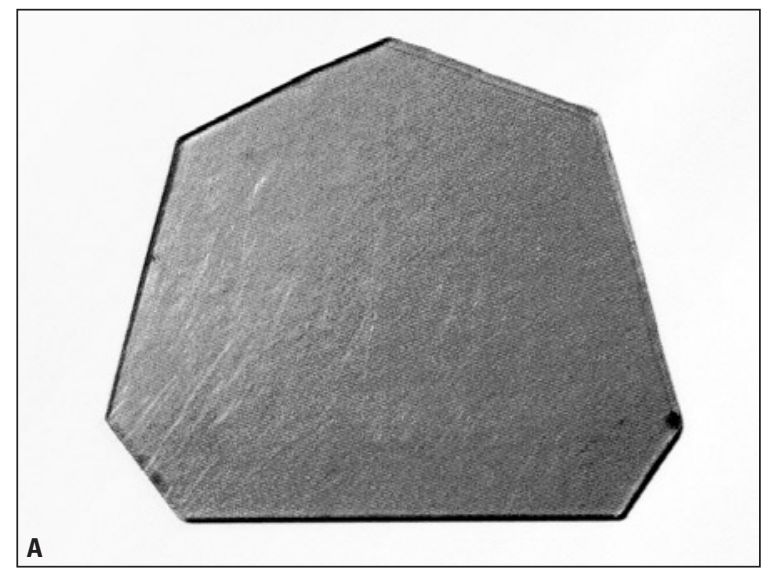

FIGURA 1 - A) Gabarito oclusal; B) Transferidor com braço de leitura regulável. superfícies oclusal e incisal ou do transferidor com a superfície vestibular dos dentes (Fig. 2).

Foram realizadas medidas de angulação e inclinação, suficientes para identificar características do posicionamento dentário que pudessem substanciar a prescrição dos braquetes a serem utilizados.

Angulação - corresponde ao ângulo entre o EVCC de cada coroa e uma linha perpendicular ao plano oclusal (Fig. 3A).

Inclinação - corresponde ao ângulo entre uma linha perpendicular ao plano oclusal e uma linha paralela e tangente ao EVCC (Fig. 3B).

Optou-se pela não inclusão dos incisivos laterais superiores e dos segundos molares. Os incisivos laterais porque, nesse tipo de fissura, freqüentemente estão ausentes ${ }^{9,11}$ ou são mal posicionados e inaproveitáveis. Os segundos molares não foram incluídos porque estavam, em grande parte dos casos, parcialmente irrompidos. Devido à dificuldade de se encontrar amostra suficiente, ou seja, encontrar pacientes que se enquadrassem em todos os requisitos, desistiu-se da sua avaliação.

As medições foram feitas, dente a dente, por um único examinador.

Em um grupo de 10 modelos, também escolhidos ao acaso, essas medidas foram repetidas, em um intervalo de uma semana, para testar o erro do método. O coeficiente de correlação de Pearson foi utilizado para avaliar esses resultados.

A avaliação estatística consistiu na obtenção

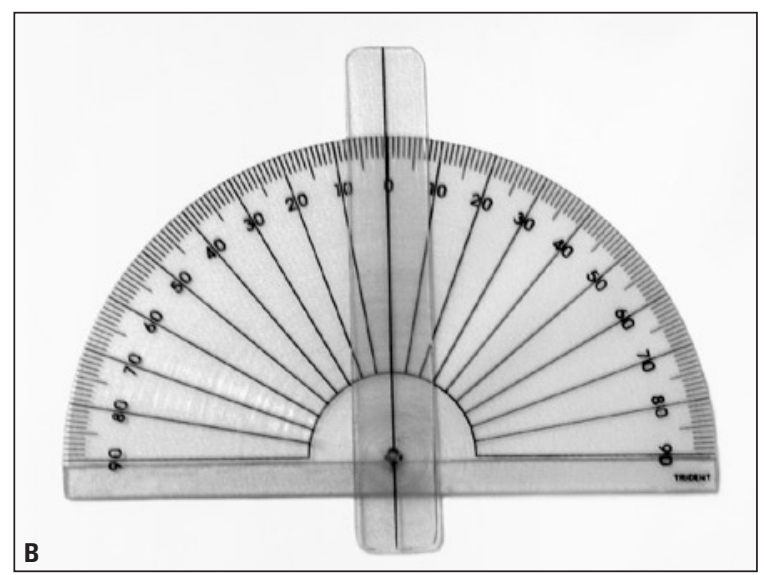


dos valores médios, desvios-padrão, medianas, máximos e mínimos, para cada elemento dentário.

\section{RESULTADOS}

Os resultados obtidos estão dispostos nas tabelas 1 a 4 .

Não houve diferença estatisticamente significante ( $p>0.05)$ entre a primeira e segunda medidas, exceto para angulação do dente 35 .

\section{Angulação}

No arco superior, foram encontrados valores positivos, com tendência crescente progressiva,

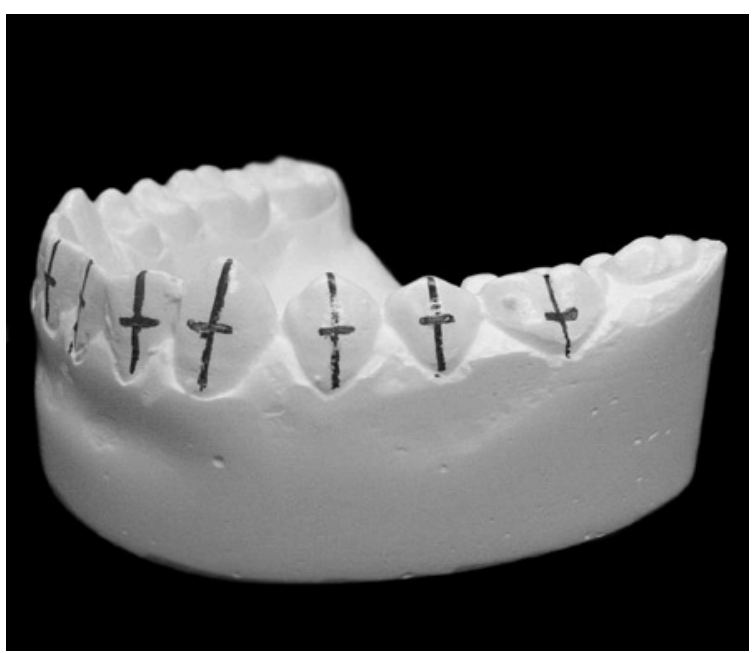

FIGURA 2 - Modelos preparados para medição, com marcações e desgastes.

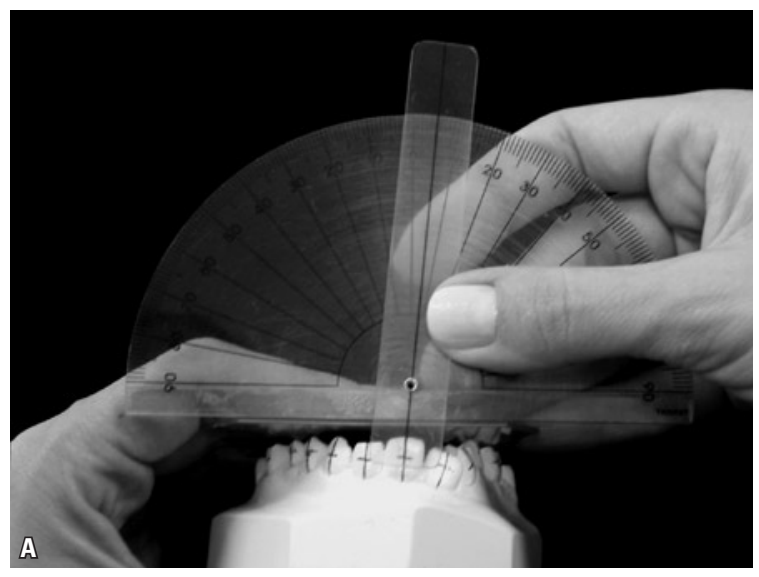

FIGURA 3 - A) Medição da angulação; B) Medição da inclinação. dos molares em direção aos caninos (área da fissura), exceção feita ao dente 25; para os incisivos, encontraram-se valores negativos, correspondentes à contra-angulação (Fig. 4).

No arco inferior, valores positivos, decrescentes de posterior para anterior (molares e pré-molares) e valores negativos, indicativos de contra-angulação, em toda a região anterior (Fig. 4).

\section{Inclinação}

Foram encontrados valores negativos, correspondentes a inclinação palatina ou lingual, para todos os dentes da amostra, superiores e inferiores.
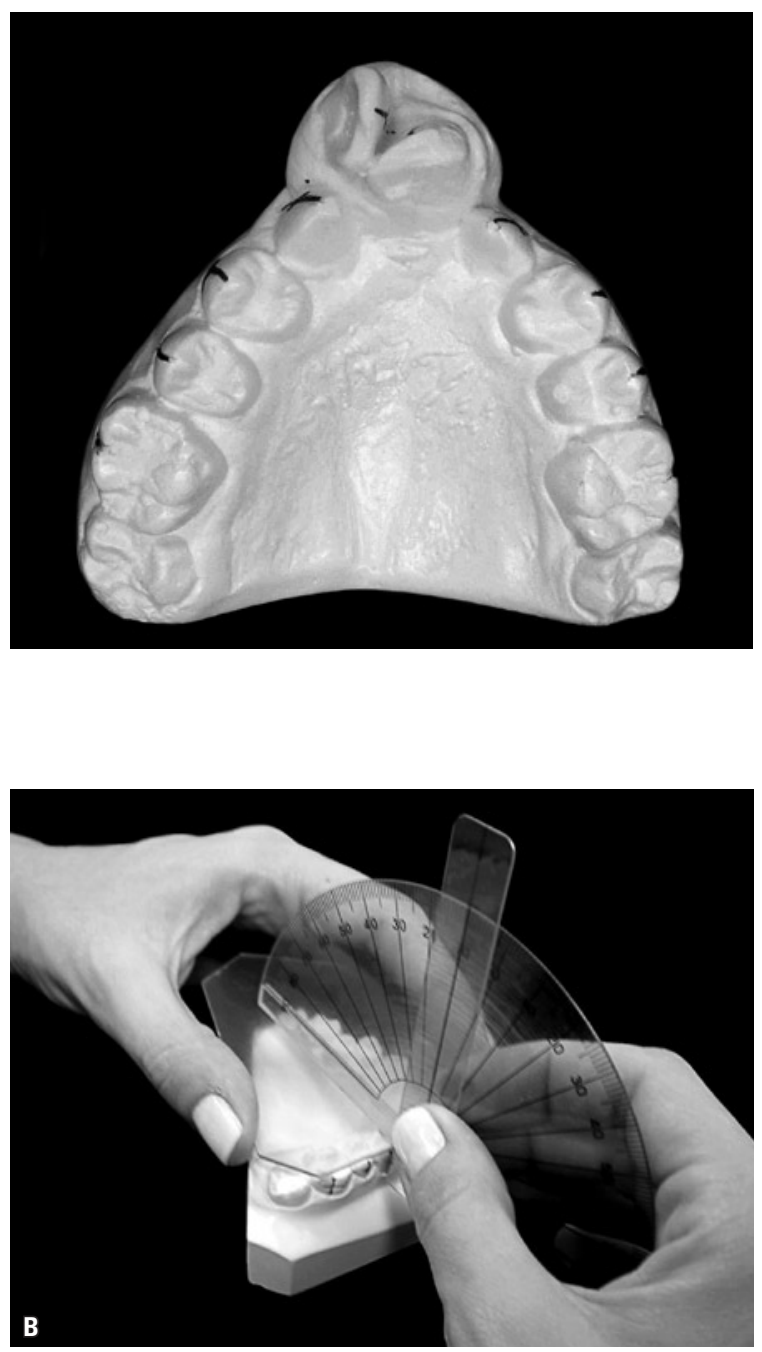
Tabela 1 - Coeficiente de correlação de Pearson: análise comparativa entre as medidas de angulação para teste do erro do método.

\begin{tabular}{|c|c|c|c|c|c|}
\hline Variáveis & Correlação & Prob. (bicaudal) & Intersecção & Coef. ang. & Número \\
\hline $16_{1} \times 16_{2}$ & 0.97 & 0.00 & -0.40 & 1.04 & 10 \\
\hline $15, \times 152$ & 0.98 & 0.00 & -0.22 & 0.93 & 8 \\
\hline $14_{1} \times 14_{2}$ & 0.98 & 0.00 & 0.35 & 0.86 & 10 \\
\hline $13_{1} \times 13_{2}$ & 0.98 & 0.00 & 1.45 & 0.89 & 9 \\
\hline $11_{1} \times 11_{2}$ & 0.98 & 0.00 & 0.01 & 0.84 & 7 \\
\hline $21_{1} \times 21_{2}$ & 0.93 & 0.00 & -0.22 & 1.06 & 10 \\
\hline $23_{1} \times 23_{2}$ & 0.98 & 0.00 & -0.20 & 0.92 & 9 \\
\hline $24_{1} \times 24_{2}$ & 0.95 & 0.00 & 0.30 & 0.86 & 10 \\
\hline $25_{1} \times 25_{2}$ & 0.95 & 0.00 & 0.28 & 0.96 & 8 \\
\hline $26_{1} \times 26_{2}$ & 0.97 & 0.00 & -0.64 & 0.95 & 10 \\
\hline $36_{1} \times 36_{2}$ & 0.85 & 0.00 & 0.23 & 0.81 & 9 \\
\hline $35_{1} \times 35_{2}$ & 0.40 & 0.29 & 1.58 & 0.53 & $9 * *$ \\
\hline $34_{1} \times 34_{2}$ & 0.89 & 0.00 & 1.18 & 0.94 & 9 \\
\hline $33_{1} \times 33_{2}$ & 0.96 & 0.00 & -0.14 & 0.94 & 10 \\
\hline $32_{1} \times 32_{2}$ & 0.98 & 0.00 & 0.05 & 0.93 & 10 \\
\hline $31_{1} \times 31_{2}$ & 0.85 & 0.00 & -0.36 & 0.63 & 10 \\
\hline $41_{1} \times 41_{2}$ & 0.98 & 0.00 & -0.37 & 1.11 & 10 \\
\hline $42_{1} \times 42_{2}$ & 0.80 & 0.00 & -1.45 & 0.81 & 10 \\
\hline $43_{1} \times 43_{2}$ & 0.94 & 0.00 & 1.23 & 0.86 & 10 \\
\hline $44_{1} \times 44_{2}$ & 0.96 & 0.00 & 0.00 & 1.00 & 10 \\
\hline $45_{1} \times 45_{2}$ & 0.90 & 0.00 & 0.16 & 0.87 & 10 \\
\hline $46_{1} \times 46_{2}$ & 0.79 & 0.01 & 2.04 & 0.52 & 10 \\
\hline
\end{tabular}

** estatisticamente signific ante

No arco superior, destacam-se valores diferentes para primeiros e segundos pré-molares e a acentuada inclinação palatina dos incisivos centrais, sendo estes últimos os maiores valores encontrados (Fig. 5).

No arco inferior, as inclinações apresentaramse acentuadamente negativas, progressivas de anterior para posterior e incisivos também com valores negativos (Fig. 5).

\section{DISCUSSÃO}

Nesse trabalho, foi estudado o posicionamento dentário em pacientes portadores de fissura trans- forame incisivo bilateral. Algumas características já descritas clínica e cefalometricamente, em trabalhos da literatura, foram aqui confirmadas, com avaliação de modelos de pacientes com fissura. Comparações serão feitas entre eles no intuito de buscar suporte ou assumir divergência, e assim o faremos por não haver relato de outro trabalho semelhante ao presente estudo.

Os resultados encontrados serão discutidos abaixo, dando maior ênfase aos valores de incisivos e caninos superiores, dentes vizinhos às áreas da fissura e, portanto, mais significativos em termos de alterações posicionais. 
Avaliação da angulação e inclinação dos elementos dentários em pacientes adultos jovens portadores de fissura transforame incisivo bilatera

Tabela 2 - Coeficiente de correlação de Pearson: análise comparativa entre as medidas de inclinação para teste de erro do método.

\begin{tabular}{|c|c|c|c|c|c|}
\hline Variáveis & Correlação & Prob. (bicaudal) & Intersecção & Coef. ang & Número \\
\hline $16_{1} \times 16_{2}$ & 0.97 & 0.00 & 0.25 & 0.97 & 10 \\
\hline $15_{1} \times 15_{2}$ & 0.97 & 0.00 & 1.20 & 0.86 & 8 \\
\hline $14_{1} \times 14_{2}$ & 0.96 & 0.00 & 3.09 & 1.11 & 10 \\
\hline $13_{1} \times 13_{2}$ & 0.97 & 0.00 & 0.08 & 0.74 & 10 \\
\hline $11_{1} \times 11_{2}$ & 0.99 & 0.00 & 0.82 & 0.95 & 7 \\
\hline $21_{1} \times 21_{2}$ & 0.99 & 0.00 & -0.45 & 0.92 & 10 \\
\hline $23_{1} \times 23_{2}$ & 0.83 & 0.00 & -0.77 & 0.69 & 10 \\
\hline $24_{1} \times 24_{2}$ & 0.98 & 0.00 & -1.81 & 0.88 & 10 \\
\hline $25_{1} \times 25_{2}$ & 1.00 & 0.00 & 0.00 & 1.00 & 8 \\
\hline $26_{1} \times 26_{2}$ & 0.89 & 0.00 & -2.60 & 0.82 & 10 \\
\hline $36_{1} \times 36_{2}$ & 0.96 & 0.00 & 2.33 & 1.11 & 9 \\
\hline $35_{1} \times 35_{2}$ & 0.97 & 0.00 & 0.07 & 0.99 & 9 \\
\hline $34_{1} \times 34_{2}$ & 0.89 & 0.00 & -2.59 & 0.87 & 9 \\
\hline $33_{1} \times 33_{2}$ & 0.89 & 0.00 & -1.91 & 0.77 & 10 \\
\hline $32_{1} \times 32_{2}$ & 0.99 & 0.00 & 3.43 & 1.09 & 10 \\
\hline $31_{1} \times 31_{2}$ & 0.98 & 0.00 & 0.77 & 0.92 & 10 \\
\hline $41_{1} \times 41_{2}$ & 0.97 & 0.00 & -1.18 & 0.85 & 10 \\
\hline $42_{1} \times 42_{2}$ & 0.98 & 0.00 & -0.65 & 0.89 & 10 \\
\hline $43_{1} \times 43_{2}$ & 0.93 & 0.00 & -0.62 & 0.90 & 10 \\
\hline $44_{1} \times 44_{2}$ & 0.96 & 0.00 & 2.51 & 1.04 & 9 \\
\hline $45_{1} \times 45_{2}$ & 0.96 & 0.00 & 9.00 & 1.33 & 10 \\
\hline $46_{1} \times 46_{2}$ & 0.95 & 0.00 & -12.15 & 0.72 & 10 \\
\hline
\end{tabular}

A inclinação palatina dos incisivos centrais superiores, com valor médio $-13^{\circ}$, está de acordo com os estudos cefalométricos ${ }^{10,16,19,20}$ e pode ser explicada como uma conseqüência da tensão muscular aumentada devido à cicatriz provocada pela queiloplastia ${ }^{10,19}$. A cirurgia pode aumentar o valor inicial da inclinação, porém esses dentes tendem a irromper retroinclinados e, na ausência de queiloplastia, podem persistir com essa inclinação ${ }^{16}$. Com relação à angulação, o valor médio encontrado de $-3^{\circ}$ talvez possa ser explicado pela ausência de tecido ósseo na distal destes dentes, o que obriga suas raízes a buscarem posicionamento mais mesial- mente. Inversamente à média, valores muito altos e positivos, indicativos de angulação excessiva dos incisivos também foram encontrados em alguns casos. Os números em si podem parecer conflitantes, mas ao observar estes modelos, verifica-se não se tratar de um desvio da angulação do dente em si, mas da própria pré-maxila como um todo, o que leva a uma análise equivocada da real angulação destes dentes, quando comparada ao plano oclusal dos segmentos posteriores (Fig. 6).

Com relação aos caninos superiores, o fato mais marcante encontrado neste estudo foi a acentuada angulação destes dentes (valores médios 18 e 19 
Tabela 3 - Valores de angulação para os diferentes elementos dentários.

\begin{tabular}{|c|c|c|c|c|c|c|}
\hline Variável & Mediana & Média & D. padrão & Mínimo & Máximo & Número \\
\hline 16 & 2 & 3.07 & 6.61 & -10 & 15 & 29 \\
\hline 15 & 5 & 5.23 & 5.31 & -3 & 15 & 26 \\
\hline 14 & 8 & 10.72 & 8.12 & 0 & 35 & 29 \\
\hline 13 & 20 & 19.12 & 9.88 & 0 & 35 & 25 \\
\hline 11 & -5 & -4.67 & 12.05 & -32 & 20 & 22 \\
\hline 21 & -2 & -2.88 & 9.43 & -28 & 24 & 25 \\
\hline 23 & 15 & 18.52 & 13.05 & 0 & 47 & 27 \\
\hline 24 & 7 & 7.64 & 6.73 & -7 & 23 & 28 \\
\hline 25 & 0 & 2.48 & 5.68 & -7 & 15 & 25 \\
\hline 26 & 4 & 3.90 & 7.08 & -8 & 20 & 30 \\
\hline 36 & 3 & 3.44 & 3.34 & 0 & 10 & 25 \\
\hline 35 & 0 & 2.78 & 6.32 & -15 & 15 & 27 \\
\hline 34 & 0 & 0.30 & 4.65 & -7 & 10 & 30 \\
\hline 33 & -2 & -2.00 & 6.04 & -15 & 10 & 29 \\
\hline 32 & 0 & -1.77 & 5.62 & -17 & 10 & 30 \\
\hline 31 & 0 & -0.53 & 3.23 & -8 & 10 & 30 \\
\hline 41 & 0 & -0.64 & 3.33 & -5 & 5 & 28 \\
\hline 42 & 0 & -0.80 & 5.25 & -15 & 11 & 30 \\
\hline 43 & -2 & -1.83 & 7.09 & -15 & 15 & 29 \\
\hline 44 & 0 & 0.86 & 5.95 & -12 & 15 & 28 \\
\hline 45 & 5 & 4.07 & 6.40 & -18 & 15 & 28 \\
\hline 46 & 5 & 5.54 & 3.75 & 0 & 15 & 26 \\
\hline
\end{tabular}

Valores negativos $=$ contra-angulação

Valores positivos $=$ angulação

graus). Algumas causas para estes valores podem ser formuladas: a primeira seria, da mesma forma que para os incisivos, o fato dos caninos estarem adjacentes à fissura; a ausência de tecido ósseo na mesial destes dentes obrigaria as raízes a buscarem uma posição mais distal, o que favoreceria uma maior angulação destes dentes. Adicionalmente, a freqüente ausência de contato proximal mesialmente ao canino, devido à agenesia do incisivo lateral, favoreceria uma migração mesial que contribuiria para esta maior angulação. E, finalmente, a deficiência alveolar vertical freqüentemente encontrada ${ }^{8}$, caracterizada pela ascendência dos segmentos maxilares nessa região, faria com que geometricamente o canino se apresentasse mais angulado em sua base, já que o gabarito oclusal utilizaria uma porção mais posterior dos segmentos maxilares para compor o plano oclusal de medição. $\mathrm{Na}$ avaliação dos modelos, pode-se verificar que, eventualmente, os caninos não estavam presentes ou estavam em posições que impediam a medição: impacção na área da fissura, persistência dos caninos decíduos antecessores (Fig. 7), irrupção na área da fissura, por palatino, ou ainda por vestibular, em infra-vestíbulo-versão.

No arco inferior foi encontrada inclinação lin- 
Avaliação da angulação e inclinação dos elementos dentários em pacientes adultos jovens portadores de fissura transforame incisivo bilateral

Tabela 4 - Valores de inclinação para os diferentes elementos dentários.

\begin{tabular}{|c|c|c|c|c|c|c|}
\hline Variável & Mediana & Média & D. padrão & Mínimo & Máximo & Número \\
\hline 16 & -10 & -10.10 & 8.92 & -25 & 10 & 29 \\
\hline 15 & -10 & -7.61 & 8.92 & -20 & 15 & 26 \\
\hline 14 & -15 & -12.41 & 9.27 & -40 & 8 & 29 \\
\hline 13 & 0 & -4.87 & 8.34 & -30 & 7 & 30 \\
\hline 11 & -14.5 & -14.09 & 13.10 & -45 & 5 & 22 \\
\hline 21 & -10 & -12.00 & 10.52 & -40 & 7 & 25 \\
\hline 23 & 0 & -4.47 & 7.28 & -20 & 10 & 30 \\
\hline 24 & -10 & -10.57 & 10.03 & -30 & 7 & 28 \\
\hline 25 & -10 & -7.00 & 8.90 & -20 & 10 & 25 \\
\hline 26 & -10 & -11.00 & 6.62 & -20 & 0 & 30 \\
\hline 36 & -40 & -37.84 & 9.79 & -60 & -20 & 25 \\
\hline 35 & -30 & -30.22 & 7.87 & -45 & -10 & 27 \\
\hline 34 & -25 & -26.90 & 8.73 & -45 & -10 & 29 \\
\hline 33 & -12 & -12.93 & 7.70 & -25 & 5 & 29 \\
\hline 32 & -5 & -7.65 & 7.41 & -25 & 6 & 29 \\
\hline 31 & -7.5 & -8.00 & 9.02 & -25 & 10 & 30 \\
\hline 41 & -5 & -7.55 & 10.71 & -40 & 10 & 29 \\
\hline 42 & -7 & -7.53 & 7.80 & -23 & 5 & 30 \\
\hline 43 & -10 & -13.10 & 6.87 & -25 & 5 & 29 \\
\hline 44 & -25 & -25.78 & 6.59 & -40 & -15 & 28 \\
\hline 45 & -30 & -31.34 & 9.63 & -45 & -5 & 29 \\
\hline 46 & -40 & -39.69 & 7.62 & -60 & -30 & 26 \\
\hline
\end{tabular}

Valores negativos = inclinação lingual ou palatina

Valores positivos $=$ inclinação vestibular

gual de todos os dentes, denotando uma possivel adaptação à forma atrésica do arco superior, pressão do lábio ou plano mandibular obtuso ${ }^{22}$. O apinhamento ântero-inferior foi um achado comum entre os casos. Os resultados do arco inferior, por não sofrerem influência direta da fissura, foram mais uniformes, comparando-se os hemiarcos.

Nota-se que para todos os dentes medidos foram obtidos valores máximos e mínimos muito discrepantes, o que mais uma vez confirma a grande variação existente entre os casos desse mesmo tipo de fissura. "Variação é a regra, não a exceção"21. Isto vale mais para magnitude que para direção.
Os segundos molares superiores, apesar de não terem sido medidos, apresentaram-se com trajeto de irrupção distal e os inferiores, mesial, na maior parte dos casos. Quanto à inclinação, assemelhavam-se à dos primeiros molares adjacentes.

A realização do estudo em fase de dentadura permanente, como era previsto, trouxe consigo algumas dificuldades como a impossibilidade de se realizar algumas medições em virtude de perda de dentes por cárie e apinhamentos (falta de espaço para irrupção de segundos pré-molares, por exemplo). Talvez isso explique a variação dos resultados entre os primeiros e segundos pré-mo- 


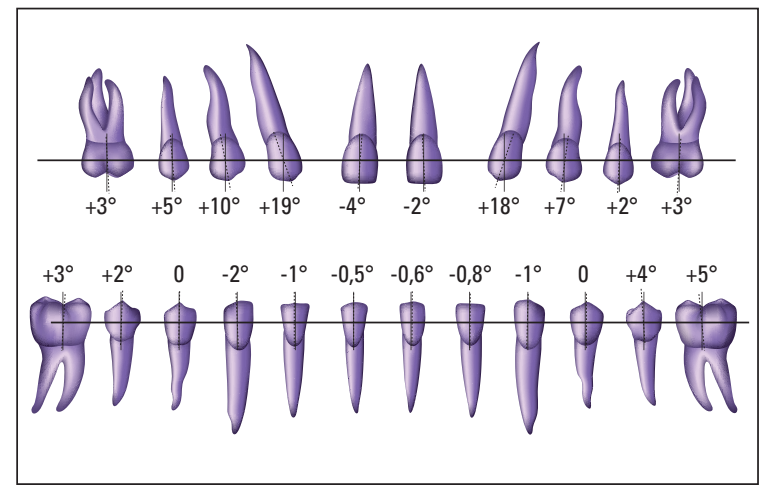

FIGURA 4 - Valores médios aproximados para angulação.

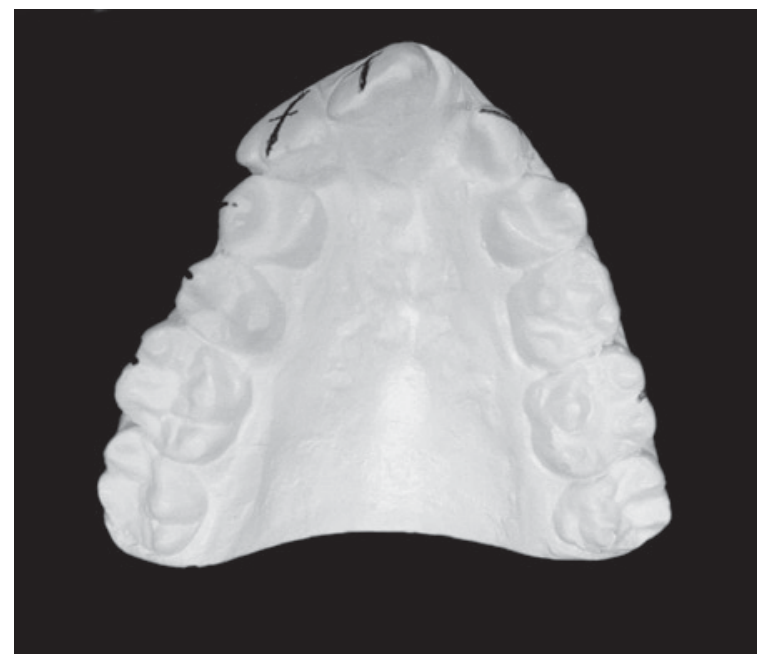

FIGURA 6 - Modelo apresentando desvio de pré-maxila.

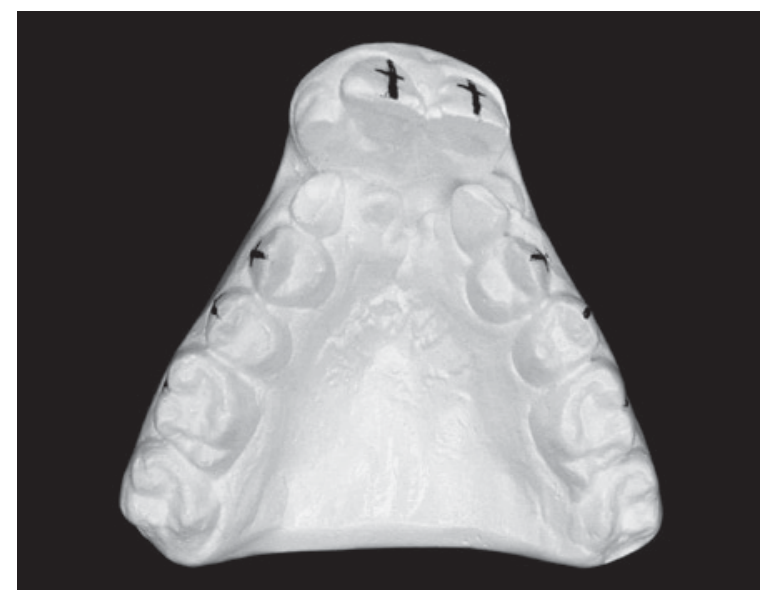

FIGURA 7 - Modelo apresentando irrupção dos caninos permanentes nas áreas de fissura e persistência dos caninos decíduos.

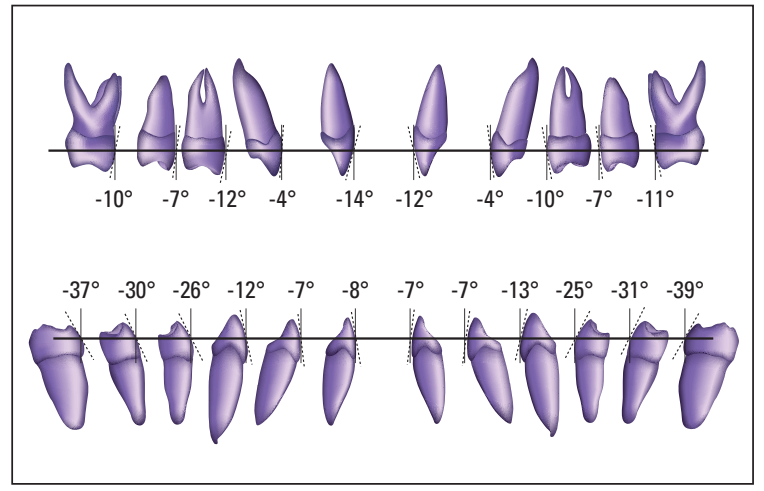

FIGURA 5 - Valores médios aproximados para inclinação.

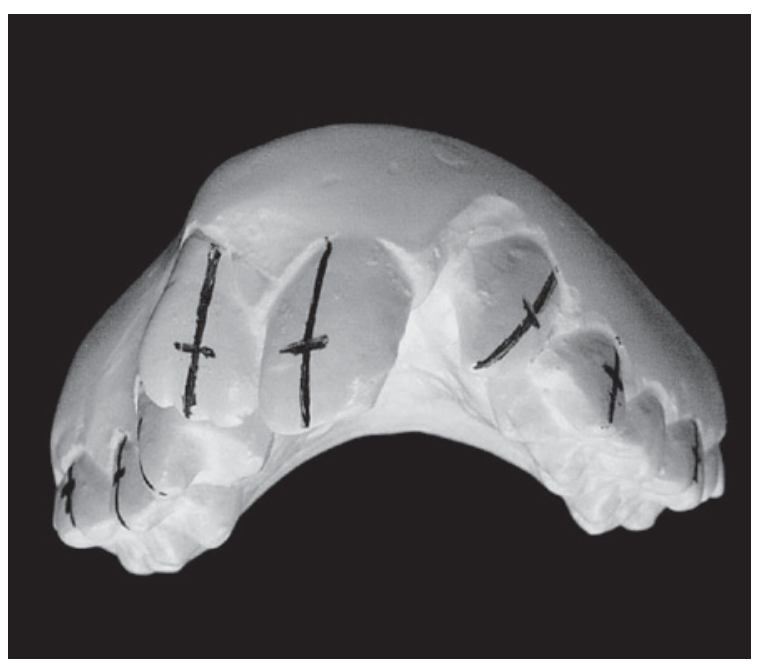

lares superiores, estando esses últimos mais verticalizados e menos inclinados. A escolha dessa fase do desenvolvimento oclusal também dificultou a seleção da amostra, uma vez que um grande número de pacientes adultos jovens avaliados já apresentavam discrepância maxilo-mandibular compatível com indicação de tratamento cirúrgico, não mais compensatório, não podendo ser incluídos na amostra.

A definição de um plano oclusal utilizando-se o gabarito acrílico também foi um dos fatores de dificuldade da metodologia. Enquanto Andrews ${ }^{1}$ trabalhou com modelos de oclusão natural perfei- 
ta, o presente estudo utilizou-se de modelos de pacientes com fissura bilateral e grande assimetria. Portanto, os resultados numéricos devem ser encarados com cautela.

No que diz respeito ao tratamento desses pacientes, a escolha de braquetes no arco superior deve ser individualizada, levando-se em consideração a presença ou não de incisivos laterais, realização de enxerto ósseo alveolar secundário na área da fissura e formato das coroas dos dentes. $\mathrm{O}$ arco inferior apresentou valores mais uniformes para angulação e inclinação sugerindo compensação à deficiência maxilar. Deste modo, a prescrição III, desenvolvida por Capelozza Filho et al. ${ }^{7}$, pode ser utilizada para manter essa compensação.

\section{CONCLUSÕES}

O presente trabalho avaliou a inclinação e angulação dos elementos dentários em pacientes portadores de fissura transforame incisivo bilateral. Os resultados encontrados mostraram grandes variações entre os indivíduos, principalmente no arco superior, devido à presença da fissura. Com base nestes resultados algumas considerações podem ser formuladas:
- Caninos e incisivos superiores foram os dentes com valores mais diferentes e variantes em relação ao esperado para amostras de oclusão normal, o que denota o grau de importância da fissura no posicionamento dos mesmos;

- O arco inferior manteve valores mais uniformes e simétricos que o arco superior, devido à menor interferência da fissura;

- As inclinações encontradas para os dentes inferiores foram todas negativas e o apinhamento nos incisivos inferiores foi um achado freqüente. Isto denota uma compensação do arco inferior à forma atrésica da maxila;

- Com relação à possibilidade de uso do aparelho programado nestes pacientes, deve-se ter critérios para individualizar a prescrição de acordo com cada caso, principalmente para o arco superior. Para o arco inferior, a prescrição III de Capelozza $\mathrm{Filho}^{7}$, pode ser recomendada na maioria dos casos, como forma de manter a compensação encontrada na maior parte dos indivíduos deste estudo.

\title{
Evaluation of tipping and inclination of teeth in young adults with complete bilateral cleft lip and palate
}

\begin{abstract}
The aim of this study was to evaluate and measure the tooth positioning (tipping and inclination) in patients with complete bilateral cleft lip and palate in an attempt to establish the features common to these patients. For that purpose, thirty pairs of dental casts were randomly selected from the files of the Hospital of Rehabilitation of Craniofacial Anomalies. The dental casts had been obtained from treated patients with this type of cleft in the permanent dentition, aged 12 to 27 years. The casts were prepared and the measurements were performed based on the method suggested by Andrews ${ }^{1}$. The results achieved were the following: 1 . Tipping - in the maxillary arch, positive values (positive tipping) with a progressively increasing tendency were observed from the molars to the canines, and negative values for the incisors (counter-tipping). As regards the mandibular arch, decreasing positive values were found from the molars to the premolars and negative values were observed for the entire anterior region; 2 . Inclination - negative values, corresponding to palatal or lingual inclination, were found for all teeth in the sample. It was concluded that the maxillary canines and incisors were the teeth most affected by the presence of the cleft: the mandibular arch kept more uniform values due to the middle influence from the cleft; the negative inclinations observed for the entire mandibular arch and the frequent anterior crowding suggest adaptation of the mandibular arch to compensate the maxillary atresia.
\end{abstract}

Key words: Orthodontics. Orthodontic brackets. Cleft lip and palate. 


\section{REFERÊNCIAS}

1. ANDREWS, L. F. Straigh wire: o conceito e o aparelho. 2. ed. Tradução por L. A. Wells. Curitiba: Produções Interativas, 1996.

2. ATHANASIOU, A. E.; MAZAHERI, M.; ZARRINNIA, K. Longitudinal study of the dental arch dimensions in bilateral cleft lip and palate patients. J. Pedod., Birmingham, v. 11, no. 3, p. 253-268, Spring 1987.

3. ATHERTON, J. D. The natural history of the bilateral cleft. Angle Orthod., Appleton, v. 44, no. 4, p. 269-278, Oct. 1974.

4. BISHARA, S. E.; OLIN, W. H.; KRAUSE, C. J. Cephalometric findings in two cases with unrepaired bilateral cleft lip and palate. Cleft Palate J., Baltimore, v. 15, no. 3, p. 233-238, July 1978

5. CAPELOZZA FILHO, L.; SILVA FILHO, O. G. Fissuras lábiopalatais. In: PETRELLI, E. Ortodontia para Fonoaudiologia. Curitiba: Lovise, 1992. p. 195-239.

6. CAPELOZZA FILHO, L.; FREITAS, J. A. S.; FREITAS, P. Z.; CAPELOZZA, J. A. Z. Ortodontia na dentadura mista em pacientes com fissura: avaliação da efetividade pelo exame da oclusão na dentadura permanente. Ortodontia, São Paulo, v. 28, n. 2, p. 71-78, maio/ago. 1995.

7. CAPELOZZA FILHO, L.; SILVA FILHO, O. G.; OZAWA, T. O.; CAVASSAN, A. O. Individualização de braquetes na técnica de straight-wire: revisão de conceitos e sugestão de indicações de uso. Rev. Dental Press Ortodon. Ortop. Facial, Maringá, v. 4, n. 4, p. 87-106, jul./ago. 1999.

8. CHAPMAN, J.H. Orthodontic treatment of children with cleft of the lip and the palate. Aust. Orthod. J., Brisbane, v. 4, no. 1, p. 17-22, Feb. 1975

9. FISHMAN, L. S. Factors related to tooth number, eruption time, and tooth position in cleft palate individual. ASDC J. Dent.

Child., Chicago, v. 37, no. 4, p. 303-306, July/Aug. 1970.

10. HANDELMAN, C. S.; PRUZANSKY, S. Occlusion and dental profile with complete bilateral cleft lip and palate. Angle Orthod., Appleton, v. 38, no. 3, p. 185-198, July 1968.

11. HEIDBÜCHEL, K. L.; KUIJPERS-JAGTMAN, A. M. Maxillary and mandibular dental-arch dimensions and occlusion in bilateral cleft lip and palate patients from 3 to 17 years of age. Cleft Palate Craniofac. J., Pittsburgh, v. 34, no. 1, p. 21-26, Jan. 1997.

12. NARULA, J. K.; ROSS, R. B. Facial growth in children with complete bilateral cleft lip and palate. Cleft Palate J., Baltimore, v. 7, p. 239-248, Jan. 1970.

13. PETERKA, M. Upper alveolar arch development in patients with total bilateral cleft lip and palate. Acta Chir. Plast., Prague, v. 26 , no. 1 , p. $30-38,1984$
14. SEMB, G. A study of facial growth in patients with bilateral cleft lip and palate treated by the Oslo CLP team. Cleft Palate Craniofac. J., Pittsburgh, v. 28, no. 1, p. 22-39, Jan. 1991.

15. SILVA FILHO, O. G.; FREITAS, J. A. S.; OKADA, T. Fissuras labiopalatais: diagnóstico e uma filosofia interdisciplinar de tratamento. In: PINTO, V. G. Saúde bucal coletiva. São Paulo: Ed. Santos, 2000. p. 481-527.

16. SILVA FILHO, O. G.; LAURIS, R. C. M. C.; CAPELOZZA FILHO, L.; SEMB, G. Craniofacial morphology in adult patients with unoperated complete bilateral cleft lip and palate. Cleft Palate Craniofac. J., Pittsburgh, v. 5, no. 2, p. 111-119, Mar. 1998.

17. SILVA FILHO, O. G.; MACHADO, F. M. C.; ANDRADE, A. C.; SOUZA FREITAS, J. A.; BISHARA, S. E. Upper dental arch morphology of adult unoperated complete bilateral cleft lip and palate. Am. J. Orthod. Dentofacial Orthop., St. Louis, v. 114 , no. 2, p. 154-161, Aug. 1998

18. SILVA FILHO, O. G.; RAMOS, A. L.; CAPELOZZA FILHO, L. Influência da queiloplastia nas dimensões e forma do arco dentário superior em adultos fissurados de lábio e palato. Rev. Soc. Brás. Ortodon., Rio de Janeiro, v. 1, n. 9, p. 269-275, abr./dez. 1991.

19. SILVA FILHO, O. G.; VALLADARES NETO, J.; CAPELOZZA FILHO, L.; SOUZA FREITAS, J. A. Influência da queiloplastia sobre a morfologia craniofacial em fissura bilateral completa de lábio e palato. Ortodontia, São Paulo, v. 34, n. 1, p. 17-26, jan./mar. 2001.

20. ŠMAHEL, Z. Craniofacial morphology in adults with bilateral complete cleft lip and palate. Cleft Palate J., Baltimore, v. 21, no. 3, p. 159-169, July 1984

21. SUBTELNY, J. D. Orthodontic treatment on cleft lip and palate birth to adulthood. Angle Orthod., Appleton, v. 36, no. 4, p. 273-292, Oct. 1966.

22. SWANSON, L. T.; MACCOLLUM, D. W.; RICHARDSON, S. O Evaluation of the dental problems in the cleft palate patient. Am. J. Orthod., St. Louis, v. 42, no. 10, p. 749-765, Oct. 1956.

23. VALE, D. M. V.; SOUZA FREITAS, J. A.; CAPELOZZA FILHO, L. Avaliação cefalométrica do crescimento maxilar e seu comportamento sob variáveis cirúrgicas, de jovens portadores de fissura transforame incisivo bilateral. Ortodontia, São Paulo, v. 26, n. 3, p. 15-25, set./dez. 1993.

24. VALE, D. M. V.; CAPELOZZA FILHO, L.; SILVA FILHO, O. G. O comportamento da mandíbula frente ao crescimento e ao tratamento cirúrgico nas fissuras transforame incisivo bilateral. Ortodontia, São Paulo, v. 27, n. 1, p. 4-12, jan./abr. 1994.
Endereço para correspondência

Leopoldino Capelozza Filho

Rua Silvio Marchione, 3-20 - Setor de Ortodontia do HRAC-USP

CEP: 17.012-900 - Bauru / SP

E-mail: Icapelozza@uol.com.br 